\title{
Phase Transition in Swollen Gels XXVIII. Swelling and Mechanical Behavior of Poly(1-vinyl-2-pyrrolidone-co- $N$-vinylcaprolactam) Gels in Water/Acetone Mixtures
}

\author{
Michal IlavskÝ, ${ }^{*},{ }^{\dagger}$ Galym MamytBekov, ${ }^{*},{ }^{\dagger \dagger}$ Zdeňka Sedláková, ${ }^{*}$ and Esen Abikenovich BeKTURov ${ }^{*} *$ \\ Faculty of Mathematics and Physics, Charles University, 18000 Prague 8, Czech Republic \\ ${ }^{*}$ Institute of Macromolecular Chemistry, Academy of Sciences of the Czech Republic, \\ 16206 Prague 6, Czech Republic \\ ** Institute of Chemical Sciences, Academy of Sciences of the Kazakh Republic, \\ Almaty, Kazakh Republic
}

(Received August 4, 2000; Accepted December 6, 2000)

\begin{abstract}
Networks of statistical copolymers of 1-vinyl-2-pyrrolidone and $N$-vinylcaprolactam (molar ratios of $\mathrm{VP} / \mathrm{VCL}=1 / 0,0.8 / 0.2,0.6 / 0.4$, and $0.5 / 0.5$ ) with ionic comonomer, $N, N$-dimethyl- $N, N$-diallylammonium chloride (mole fractions $x_{\mathrm{S}}=0,0.03$, and 0.05 ) and crosslinker, 1-1'-divinyl-3,3'-(ethane-1,1-diyl)di(1-vinyl-2-pyrrolidone), were prepared by radiation polymerization in a water/ethanol mixture $\left(\mathrm{H}_{2} \mathrm{O} / \mathrm{EtOH}=0.5 / 0.5\right.$ by vol. $)$. Swelling and mechanical behavior was investigated in water/acetone (w/a) mixtures. For charged copolymers, a first-order phase transition (collapse) was found. The extent of collapse (stepwise change in the gel volume), $\Delta$, and critical acetone concentration in the mixture at collapse, $a_{\mathrm{c}}$, slightly increase with content of VCL and ammonium salt in gels. Shift of transition to higher $a_{\mathrm{c}}$ is caused by increasing hydrophobicity of network chains with VCL content. Decrease in swelling with increasing acetone concentration in w/a mixtures is accompanied by increase in equilibrium modulus, so that mechanical behavior is predominantly determined by swelling. The theory of swelling equilibria of polyelectrolyte networks, in which the effects of electrostatic interactions of the charges on the chain and finite chain extensibility are included, semiquantitatively describes the swelling data provided an effective concentration of charges (lower than $x_{\mathrm{s}}$ ) was introduced.

KEY WORDS First-Order Phase Transition / 1-Vinyl-2-pyrrolidone / N-Vinylcaprolactam Copolymers / Polyelectrolytes / Swelling Equilibria / Mechanical Behavior / Collapse Phenomenon /
\end{abstract}

One advantage of hydrogels consists in capability of high swelling and exhibiting first-order phase transition (collapse) caused by small change in external conditions such as temperature, electric field, solvent compositions etc. ${ }^{1}$ The presence of charges on the chain $(\sim 1-10$ mol\%) seems to be an important condition for the occurrence of a jump change in gel volume. ${ }^{2-4}$ Most results were obtained with charged networks of polyacrylamide (gels sensitive to solvent composition) or with networks of ionic poly $(N, N$-diethylacrylamide) and charged poly ( $N$-isopropylacrylamide) (temperature sensitive hydrogels). This transition, in particular temperature-induced collapse, can be widely used. ${ }^{5}$

Progressive interest in hydrogels based on poly(1vinyl-2-pyrrolidone) (PVP) and copolymers can be found in recent literature. ${ }^{6-8}$ This interest is associated with unique properties of PVP systems (e.g., PVP forms reversible association complexes with dyes or some reactive polymers ${ }^{9-11}$ ). PVP gels have applications in biomedical devices, agriculture, and many others. Our previous papers report ${ }^{12}$ swelling and mechanical behavior of positively charged networks of 1-vinyl-2-pyrrolidone in water/acetone mixtures. In the hydrogels, collapse was found at critical acetone concentrations $\alpha_{\mathrm{c}} \sim 75 \mathrm{vol} \%$; the extent of collapse (stepwise change in the gel volume), $\Delta$, and $a_{\mathrm{c}}$ increased with charges bound to the chain.

Copolymerization of two monomers is an effective method of modification of physical properties of gels. Several authors, ${ }^{13-15}$ who have studied temperature col- lapse in $\operatorname{poly}(N$-isopropylacrylamide) hydrogels, report that while incorporation of a hydrophilic comonomer leads to higher transition temperature (LCST), incorporation of a hydrophobic monomer lowers transition temperature. This work investigates the effects of composition of ionized networks of statistical copolymers of 1 vinyl-2-pyrrolidone)/ $N$-vinylcaprolactam (VP/VCL) prepared by radiation polymerization in water/ethanol mix ture $\left(\mathrm{H}_{2} \mathrm{O} / \mathrm{EtOH}=0.5 / 0.5\right.$ by vol. $)$ on swelling and mechanical behavior in water/acetone (w/a) mixtures at room temperature. VCL is a more hydrophobic component due to the higher number of $\mathrm{CH}_{2}$ groups than in VP.

\section{EXPERIMENTAL}

\section{Materials}

Purification of 1-vinyl-2-pyrrolidone (VP) and $N$ vinylcaprolactam (VCL, Fluka) was described previously. ${ }^{12,14} \mathrm{~N}, N$-dimethyl- $N, N$-diallylammonium chloride (Fluka, 65 vol\% aqueous solution of monomer/water)<smiles>C=CC[N+](C)(C)CC=C</smiles>

was used as ionic comonomer. The crosslinker used was 1-1' - divinyl-3,3' - (ethane-1,1-diyl) di (1-vinyl-2pyrrolidone)

\footnotetext{
${ }^{\dagger}$ To whom correspondence should be addressed (Phone: +420-2-21912363, Fax: +420-2-6885095, E-mail: ilavsky@kmf.troja.mff.cuni.cz).

${ }^{\dagger \dagger}$ Participant of the UNESCO-sponsored postgraduate course. On leave from the Academy of Sciences of the Kazakh Republic.
} 
<smiles>C=CN1CCC(C(C)C2CCN(C=C)C2)C1</smiles>

synthesized as shown previously. ${ }^{17}$

\section{Network Preparation}

Four networks, copolymers of 1-vinyl-2-pyrrolidone and $N$-vinylcaprolactam (molar ratios of $\mathrm{VP} / \mathrm{VCL}=1 / 0$, $0.8 / 0.2,0.6 / 0.4$, and $0.5 / 0.5$ ) with various amounts of ammonium salt $\left(\mathrm{S}\right.$, mole fraction, $x_{\mathrm{s}}=0,0.03$, and 0.05 , Table I) and constant amount of the crosslinker (mole fraction $x_{\mathrm{CR}}=0.03$ ) were prepared by $\gamma$-radiation copolymerization within water/ethanol mixture $(0.5 / 0.5$ by vol.). The volume fraction of mixture of monomers was $v_{\mathrm{m}}=$ 0.1 . Glass ampoules $10 \mathrm{~mm}$ in diameter were used as polymerization reactors and required radiation doses shown in Table I ( $\gamma$-source $\mathrm{Co}^{60}$ with a dose rate of 0.8 $\mathrm{kGy} \mathrm{h}^{-1}$, Artim Prague) were applied at room temperature $\left(25^{\circ} \mathrm{C}\right)$. Due to high diluent content at polymerization, the effect of the reaction heat was negligable $(<$ $\left.1{ }^{\circ} \mathrm{C}\right)$.

After polymerization, the copolymers were removed from ampoules, cut into pieces $10 \mathrm{~mm}$ in length and extracted with redistilled water. Some samples $\sim 0.80 \mathrm{~cm}^{3}$ in volume, from all four series were weighed and extracted with a $10 \mathrm{~cm}^{3}$ of water. After extraction, the samples were dried in vacuum at $90^{\circ} \mathrm{C}$ to constant weight. By comparison of dry weight with theoretical dry weight, it was found that in all samples, sol fractions were less than $0.5 \mathrm{wt} \%$. This means that all the monomers were bound in the network structure. The same conclusions were obtained from mercurimetric titration (with 0.001 $\mathrm{M}$ solution of $\left.\mathrm{Hg}\left(\mathrm{ClO}_{4}\right)_{2}\right)$ of extracted solutions. Virtually no ammonium salt was present in the solutions.

\section{Swelling and Mechanical Measurements}

After extraction, the samples were immersed in water/ acetone mixtures and swelling proceeded for one month. The inverse swelling ratio $X$, relative to the network formation state, was calculated from ${ }^{4}$

$$
X=\left(D^{*} / D\right)^{3}=V^{*} / V
$$

where $D^{*}$ and $D$, respectively, are sample diameters after preparation and swelling in water/acetone mixtures, $V^{*}$ and $V$ are corresponding sample volumes. Diameters were measured with a travelling microscope (Abbe comparator). From $X$, the volume fraction of dry polymer in the swollen state $v_{2}=v_{1} X$ (where $v_{1}=v_{\mathrm{m}} / \rho$ is the volume fraction of dry copolymer network at network formation, $\rho$ is the dry density; $\rho=1.17,1.18,1.19$, and $1.20 \mathrm{~g} \mathrm{~cm}^{-3}$ for molar ratios of VP/VCL $=1 / 0,0.8 / 0.2,0.6 /$ 0.4 , and $0.5 / 0.5$, respectively) or the swelling degree relative to the dry state, $Q=1 / v_{2}$, were determined. From $X$, the reduced swelling ratios,

$$
X_{\mathrm{r}}=X_{\mathrm{w}} / X
$$

were determined $\left(X_{\mathrm{w}}\right.$, inverse swelling ratio in pure water).

Mechanical experiments, at given water/acetone concentrations, were carried out in uniaxial compression. The specimen (10 $\mathrm{mm}$ high) was compressed to a ratio $\lambda$ ( $\lambda=l / l_{0}$ where $l$ and $l_{0}$, respectively, are compressed and initial heights) and force $f$ was measured after 30-s relaxation (sufficient time for attaining equilibrium). Usually ten values of $\lambda$ and $f$ were determined $(0.8<\lambda<1)$. The shear modulus $G$ was calculated from, ${ }^{4}$

$$
G=f /\left[S_{0}\left(\lambda^{2}-\lambda^{-1}\right)\right]
$$

where $S_{0}$ is initial cross-section of the specimen.

Mechanical experiments were also done just after network preparation at room temperature (modulus $G_{1}$, Table I). From $G_{1}$, the concentration of elastically active network chains (EANCs) relative to the dry state $v_{\mathrm{d}}$ was determined from ${ }^{4}$

$$
v_{\mathrm{d}}=G_{1} /\left(R v_{1} T_{1}\right)
$$

where $R$ is the gas constant and $T_{1}=298 \mathrm{~K}$, temperature of measurement (Table I). From moduli $G$ and $G_{1}$, the reduced modulus,

$$
G_{\mathrm{r}}=G / G_{1}
$$

\begin{tabular}{|c|c|c|c|c|c|c|c|}
\hline VP/VCL & $\frac{\gamma}{\mathrm{kGy}}$ & $x_{\mathrm{s}}$ & $\frac{G_{1}}{\mathrm{~g} \mathrm{~cm}^{-2}}$ & $\frac{v_{\mathrm{d}} \times 10^{5}}{\mathrm{~mol} \mathrm{~cm}^{-3}}$ & $Q_{\mathrm{w}}$ & $\phi$ & $\chi$ \\
\hline $1.0 / 0.0$ & 2 & 0 & 53.5 & 2.49 & 45.7 & - & 0.492 \\
\hline $0.8 / 0.2$ & 2 & 0 & 47.6 & 2.21 & 52.4 & - & 0.495 \\
\hline $0.6 / 0.4$ & 2 & 0 & 47.3 & 2.20 & 51.0 & - & 0.496 \\
\hline $0.5 / 0.5$ & 2 & 0 & 37.4 & 1.77 & 63.3 & - & 0.498 \\
\hline $1.0 / 0.0$ & 5 & 0.03 & 47.5 & 2.21 & 87.7 & 0.22 & - \\
\hline $0.8 / 0.2$ & 5 & 0.03 & 48.7 & 2.28 & 87.8 & 0.24 & - \\
\hline $0.6 / 0.4$ & 5 & 0.03 & 41.6 & 1.96 & 100.0 & 0.23 & - \\
\hline $0.5 / 0.5$ & 5 & 0.03 & 35.1 & 1.66 & 107.9 & 0.21 & - \\
\hline $1.0 / 0.0$ & 5 & 0.05 & 51.0 & 2.37 & 104.2 & 0.17 & - \\
\hline $0.8 / 0.2$ & 5 & 0.05 & 48.8 & 2.28 & 115.0 & 0.18 & - \\
\hline $0.6 / 0.4$ & 5 & 0.05 & 43.2 & 2.04 & 119.0 & 0.18 & - \\
\hline $0.5 / 0.5$ & 5 & 0.05 & 37.9 & 1.80 & 120.2 & 0.16 & - \\
\hline
\end{tabular}

was calculated.

Table I. Composition, swelling, and mechanical parameters of poly(1-vinyl-2-pyrrolidone-co- $N$-vinylcaprolactam) (VP/VCL) hydrogels

$\gamma$, Radiation dose; $x_{\mathrm{s}}$, molar fraction of ionic salt; $G_{1}$, modulus after preparation; $v_{\mathrm{d}}$, crosslinking density related to the dry state; $Q_{\mathrm{w}}$, swelling degree in water (relative to the dry state) and $\phi$, correction factor. 


\section{RESULTS AND DISCUSSION}

\section{Effect of Network Formation Conditions on Their Struc- ture}

The synthesis of the VP/VCL copolymer network was carried out in water/ethanol mixture as it was not possible to prepare homogeneous aqueous solutions with higher VCL and low initial volume fraction of monomers $\left(v_{\mathrm{m}}=0.1\right)$. The ethanol present in the polymerization mixture may cause better solubility of growing polymer molecules in mixed water/EtOH diluent and homogeneous networks may form. From Table I, the initial modulus, $G_{1}$, after preparation at constant radiation dose $\gamma$ decreases with VCL content regardless of ionic comonomer $x_{\mathrm{s}}$. Table I shows that $G_{1}$ at constant VP/VCL composition is virtually constant for all three series even though uncharged networks form at lower dose $(\gamma=2$ $\mathrm{kGy}$ ). The efficiency of radiation crosslinking thus decreases with content of VCL. The dependence of modulus $G_{1}$ on $x_{\mathrm{s}}$ suggests that ionic comonomer, though having two double bonds, does not act as a crosslinker. Butler et $a l{ }^{18}$ found that polymerization of diallylammonium salts (S type) in water leads to the formation of linear cyclic structures ${ }^{12,18,19}$

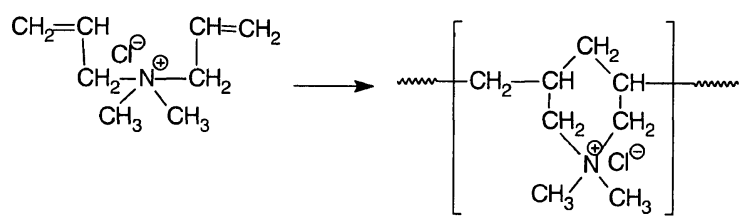

which are incorporated into the chain and finally positively charged chains are formed. Similar results were obtained $^{12}$ for pure poly (1-vinyl-2-pyrrolidone ) crosslinked with the same crosslinker (CR) by irradiation. $G_{1}$ independence of $\gamma$ (for $\gamma \geq 5 \mathrm{kGy}$ ) and decrease in $G_{1}$ with increasing $x_{\mathrm{s}}$ at constant $\gamma$ were observed. Decrease in $G_{1}$ with $x_{\mathrm{s}}$ was interpreted by transfer reaction followed by isomerization and ring closure of the radicals. ${ }^{12}$ From decrease in $G_{1}$ with VCL content in our networks (Table I) the extent of transfer reaction increases with VCL concentration.

\section{Swelling and Mechanical Behavior}

From Figure 1a, swelling $Q_{\mathrm{w}}$ in water slightly increases with VCL comonomer in all three series of networks. This increase is associated mainly with decrease in elastically active network chains (EANCs) $v_{\mathrm{d}}$ (determined from modulus $G_{1}$ ) observed in individual series (Table I). Swelling $Q_{\mathrm{w}}$ strongly increases with charged groups, $x_{\mathrm{s}}$.

Continuous or jump change in the volume of the gel (or reduced swelling ratio $X_{\mathrm{r}}$ ) with increasing volume. fraction of acetone, $a$, in the water/acetone mixtures can be seen in Figure 2. Incorporation of VCL, the more hydrophobic component, into networks results in shift of the transition region from the expanded (low $a$ values) to collapsed (high $a$ ) state to higher acetone concentrations in all three series. For uncharged networks, acetone content lies in the range from $\sim 70$ to $80 \mathrm{vol} \%$, for charged copolymers the latter is from $\sim 76$ to 86 vol\%.

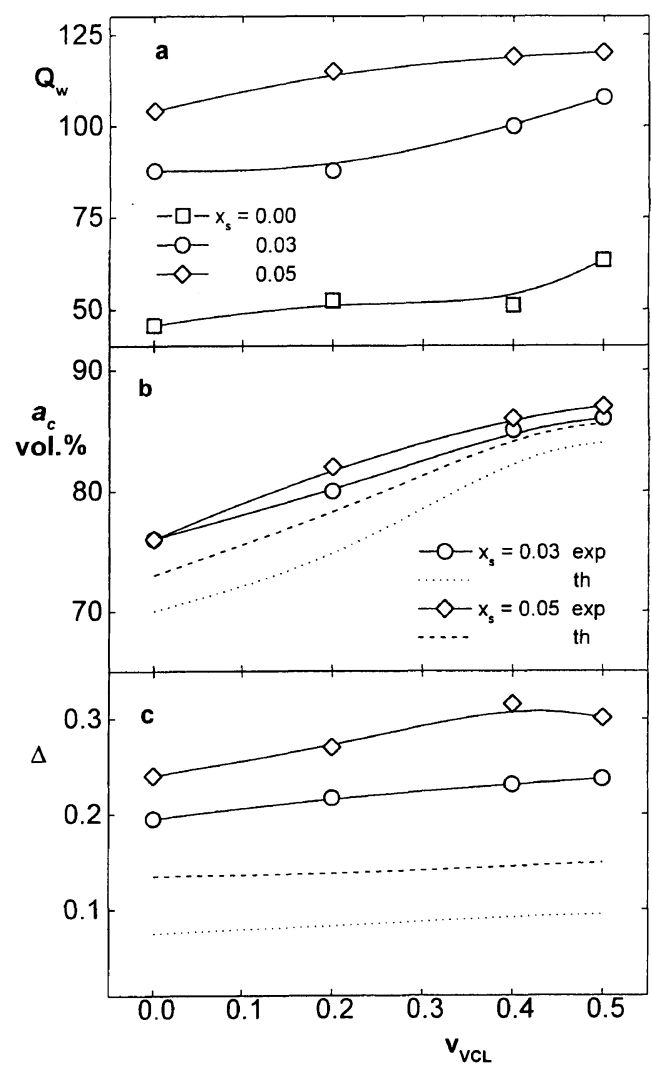

Figure 1. Dependence of swelling in water $Q_{\mathrm{w}}$, jump in volume at collapse $\Delta$ and critical acetone concentration at collapse $a_{\mathrm{c}}$ on concentration of VCL, $\mathrm{v}_{\mathrm{VCL}} \cdot \bigcirc, \diamond, \square$ experiment; - - - - theory (eq 12)

This indicates that with increasing VCL in networks, hydrogen bonding decreases and acetone becomes a better solvent for gels. In accord with this, no transition was observed earlier ${ }^{12,20}$ for uncharged poly ( $N$ vinylcaprolactam) (PVCL) gels in water/acetone mixtures. The swelling of PVCL gels in water and acetone was roughly the same.

From Figure 2, jump change in $X_{\mathrm{r}}$ takes place in networks with $x_{\mathrm{s}} \geq 0.03$. Figure $1 \mathrm{~b}$ shows that the critical acetone concentrations, at which collapse appeared, $a_{\mathrm{c}}$, are practically independent of charge concentration in the ionized series and increase with VCL content. Jump in the gel volume characterized by $\Delta\left(=v_{2}{ }^{\prime \prime}-v_{2}^{\prime}\right.$, where $v_{2}^{\prime}$ is the volume fraction of dry polymer in the expanded state and $v_{2}^{\prime \prime}$ is the volume fraction after the collapse; see inset in Figure 4), increases with $x_{\mathrm{s}}$ and does not depend on VCL content (Figure 1c).

The dependence of the reduced shear modulus, $G_{\mathrm{r}}=G /$ $G_{1}$ (eq 5) on acetone concentration $a$ in water/acetone mixtures is shown in Figure 2. As expected, jump in the volume of the gel is reflected in jump change in the reduced shear modulus. For copolymers with $x_{\mathrm{s}}=0$, continuous dependence of $X_{\mathrm{r}}$ on $a$ is accompanied by continuous dependences of modulus $G_{\mathrm{r}}$ on $a$.

The dependence of the reduced modulus, $\log G_{\mathrm{r}}$ on $\log$ $X$ for four series of copolymers with constant VP/VCL compositions are plotted in Figure 3. For comparison, the predicted rubber elasticity slope ${ }^{21}$ for the dependence of $\log G_{\mathrm{r}} v s . \log X, s=0.33$, is shown. In the range of high swelling (log $X<-0.75)$, the pronounced increase in modulus $G_{\mathrm{r}}$ with swelling can be seen for all net- 


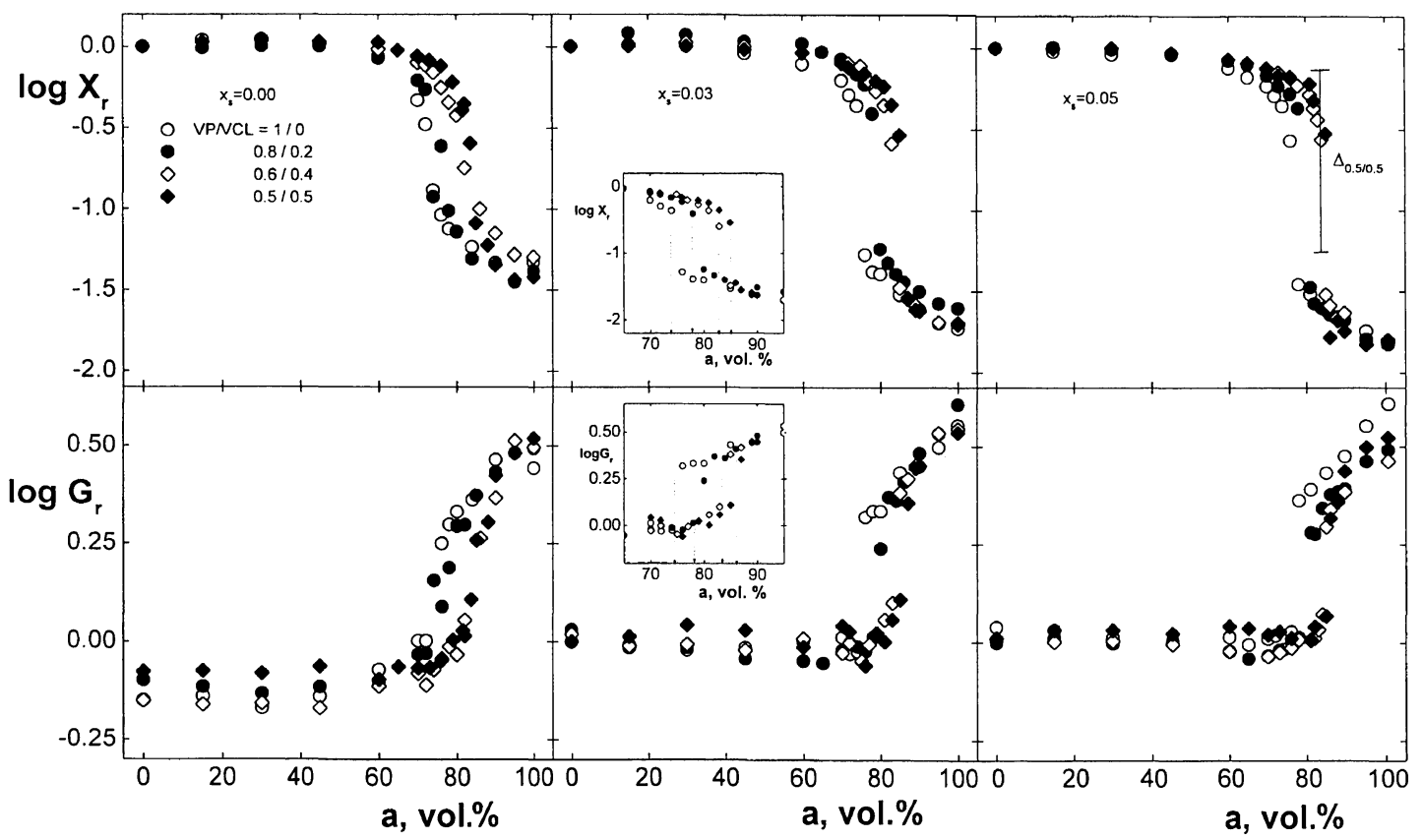

Figure 2. Dependence of reduced swelling ratio $X_{\mathrm{r}}=X_{\mathrm{w}} / X$ and reduced modulus $G_{\mathrm{r}}=G / G_{1}$ on acetone concentration $a$ for indicated VP/ VCL compositions and $x_{\mathrm{s}}$ values.

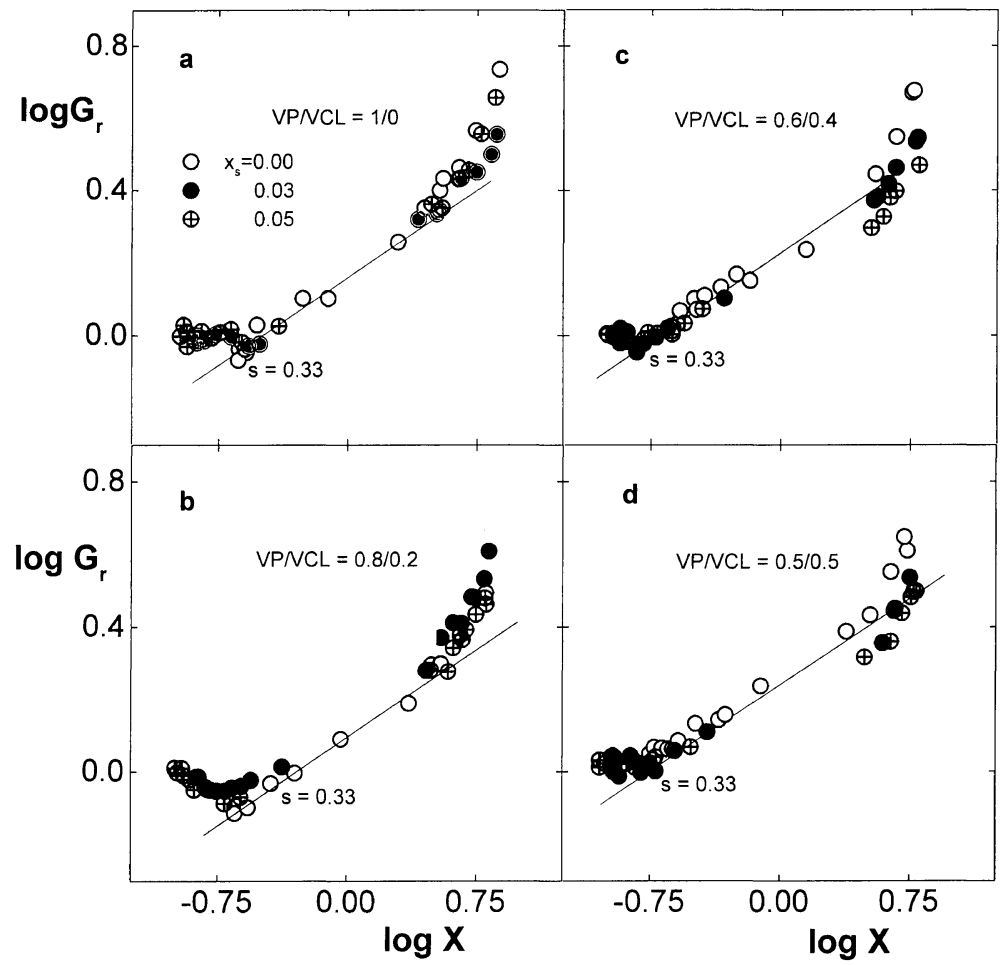

Figure 3. Dependence of reduced modulus $G_{\mathrm{r}}=G / G_{1}$ on inverse swelling ratio $X$ for indicated VP/VCL compositions and $x_{\mathrm{s}}$. Slope $s=0.33$ corresponds to the rubber elasticity theory.

works. This increase is probably due to the effects of topological constraints of high extended chains. The finite extensibility of the chains may contribute to this increase. Departures from the straight line in the region of low swelling (log $X>0.5)$ are probably due to the influence of the main transition region (vitrification) at high acetone concentrations. ${ }^{4} s=0.33$, independent of charge concentration, was also found ${ }^{12}$ for charged poly(1-vinyl- 2-pyrrolidone) networks. High $s=0.7$, independent of charge concentration, was found ${ }^{22}$ for charged polyacrylamide (PAAm) networks in water/acetone mixtures. The swelling dependence of the modulus with the slope $s$ $=5 / 6$ was recently suggested by Rubinstein et al. ${ }^{23}$ based on the scaling concept of the polyelectrolyte chain. 
Comparison of Swelling Data with the Theory of Polyelectrolyte Networks

Several models have been suggested for the swelling of charged gels. ${ }^{1-3}$ For comparison of swelling results with theory, we use our generalized model in which the effects of repulsion of charges on the chain and finite chain extensibility are included. ${ }^{3}$ In this model, all molecular parameters are independently accessible. This theory semiquantitatively describes collapse in water/ acetone mixtures and aqueous $\mathrm{NaCl}$ solutions of various charged gels. ${ }^{4,12,16}$ The theory predicts swelling pressure $P$ as the sum of four terms ${ }^{3}$

$$
P=\mu_{1} / V_{1}=P_{\mathrm{m}}+P_{\mathrm{os}}+P_{\mathrm{el}}+P_{\mathrm{els}}
$$

where $\mu_{1}$ is the chemical potential of the solvent and $V_{1}$ is molar volume of the solvent.

The contribution $P_{\mathrm{m}}$ corresponds to mixing of polymeric segments with solvent (Flory-Huggins equation)

$$
P_{\mathrm{m}}=-\left(R T / V_{1}\right)\left[\ln \left(1-v_{2}\right)+v_{2}+\chi v_{2}^{2}\right]
$$

where $\chi$ is the interaction parameter.

The contribution $P_{\text {os }}$ corresponds to the difference between osmotic pressure in the gel and solution (mixing of ions with the solvent). We consider that polyelectrolyte gel is immersed in a solution of a uni-univalent salt (e.g., $\mathrm{NaCl}$ solution) so that, ${ }^{3}$

$$
P_{\mathrm{os}}=\left(R T / M_{0}\right)\left\{i p v_{2}-2 f_{-} c_{-} M_{0}\left[\left(1+\frac{i p v_{2}}{M_{0} f_{-} c_{-}}\right)^{1 / 2}-1\right]\right\}
$$

where $M_{0}$ is molecular weight of the monomeric unit, $i$ molar ratio of the number of charges bound to the chain to number of monomeric units (ionization) and $c_{-}$and $f_{-}$, respectively, are concentrations of co-ions and activity coefficient (for $f_{-}$we use $f_{-}=0.66-0.13 \log c$, but the highest admissible value is $f_{-}=1$, see ref 3 ).

The contribution $P_{\mathrm{el}}$ was calculated from change in the configurational free energy with swelling. Owing to the high values of swelling, finite extensibility of chains had to be considered, and $P_{\mathrm{el}}$ was divided into the Gaussian, $P_{\mathrm{el}}^{\mathrm{G}}$, and non-Gaussian, $P_{\mathrm{el}}^{\mathrm{NG}}$, contributions

$$
\begin{aligned}
P_{\mathrm{el}} & \left.=P_{\mathrm{el}}^{\mathrm{G}}+P_{\mathrm{el}}^{\mathrm{NG}}=-v_{d} R T\left\langle\alpha_{0}^{2}\right\rangle^{2} v_{2}^{1 / 3}-v_{2} / 2\right) \\
& -v_{d} R T\left(\frac{3}{5}\left\langle\alpha_{0}^{2}\right\rangle^{2} v_{2}^{-1 / 3} n^{-1}+\frac{99}{175}\left\langle\alpha_{0}^{2}\right\rangle^{3} v_{2}^{-1} n^{-2}\right. \\
& \left.+\frac{513}{875}\left\langle\alpha_{0}^{2}\right\rangle^{4} v_{2}^{-5 / 3} n^{-3}+\cdots\right)
\end{aligned}
$$

where $\left\langle\alpha_{0}^{2}\right\rangle$ is the dilatation factor of the dry state $\left(\left\langle\alpha_{0}^{2}\right\rangle\right.$ $=v_{1}^{2 / 3}$ ) and $n$, number of statistical segments in the chain between crosslinks (usually expressed ${ }^{3,4}$ as the number of monomeric units in the statistical segment $s$ ).

The last contribution $P_{\text {els }}$ is determined from change in free energy of electrostatic interaction with swelling,

$$
P_{\mathrm{els}}=\frac{v_{d} N_{4} Z^{2} i^{2} e^{3} v_{2}^{4 / 3}}{3 D\left(\overline{h_{0}^{2}}\left\langle\alpha_{0}^{2}\right\rangle\right)^{1 / 2}}\left[\frac{2.5 \bar{A}}{1+\bar{A}}-\ln (1+\bar{A})\right]
$$

where $\bar{A}=6 h / \kappa \overline{h_{0}^{2}}=\left[\frac{9\left\langle\alpha_{0}^{2}>v_{2}^{-2 / 3} D k T M_{0}\right.}{\pi N_{A} e^{2} \overline{h_{0}^{2}}\left(2 M_{0} c_{-}+i \rho v_{2}\right)}\right]^{1 / 2}$

and $h$ and $\left(\overline{h_{0}^{2}}\right)^{1 / 2}$ are chain end distances in deformed and reference states, $\kappa$ is the inverse of the Debye radius of ion atmosphere, $D$, dielectric constant of medium, $k$ Bolzmann constant, $N_{A}$ Avogadro number, $Z$ degree of polymerization of the chain and $e$, unit charge (further details see ref 3 ). Swelling equilibrium for free swelling is given by,

$$
P_{\mathrm{m}}+P_{\mathrm{os}}+P_{\mathrm{el}}^{\mathrm{G}}+P_{\mathrm{el}}^{\mathrm{NG}}+P_{\mathrm{els}}=0
$$

Individual terms $P_{\mathrm{i}}$ of eqs 6 and 11 may thus be expressed in network molecular parameters: ${ }^{3}$ concentration of elastically active network chains $v_{\mathrm{d}}$ (Table I), density of polymer $\rho$, degree of ionization $i=x_{\mathrm{s}}$, average molecular weight of monomer units, $M_{0}$ molar volume of water/acetone mixture $V_{1}$ (see ref 24), volume fraction of dry polymer at network formation $v_{1}$ and permittivity of water/acetone mixtures, $D$. Using eq 11, dependence of the interaction parameter $\chi$ on volume fraction of dry polymer in the swollen state, $v_{2}=v_{1} X$, can be calculated from experimental equilibrium $X$ measured in water/ acetone mixtures (as pure water/acetone mixtures were used, the concentrations of co-ions $c_{-}=0$ ).

As expected, swelling in water $Q_{\mathrm{w}}$ increases with charged groups in chain $x_{\mathrm{s}}$ (Table I, Figure 1). Eq 11 used for uncharged case $\left(i=x_{\mathrm{s}}=0, P_{\mathrm{os}}=P_{\text {els }}=0\right)$ gives roughly constant interaction parameter $\chi=0.492$ 0.498 (Table I) regardless of network composition (slight increase in $Q_{\mathrm{w}}$ with VCL is mainly due to decreased crosslinking density). From $X$ for uncharged networks measured in various water/acetone mixtures (Figure 2), the dependence of $\chi$ on $v_{2}$ was obtained (Figure 4). Eq 11 for ionic networks with ionization $i=x_{\mathrm{s}}$, gives unrealistically high $\chi$ calculated from $Q_{\mathrm{w}}$ measured in water (for networks of various compositions with $x_{\mathrm{s}}=0.03$ and 0.05 , $\chi$ in the region $0.650-0.700$ and $0.820-0.850$ was calculated). As mentioned in theory, $\chi$ is a measure of polymer-water interactions when all charges are screened (effect of charges is included in $P_{\mathrm{os}}$ and $P_{\text {els }}$ terms), $\chi$ shown in Table I for uncharged networks may thus be, in the first approximation, required for corresponding ionic hydrogels swollen in pure water. With this requirement, $Q_{\mathrm{w}}$ shown in Figure 1 can be described by eq 11 assuming that effective ionization $i^{*}$ is lower than the salt concentration, i.e., $i^{*}=i \phi=\phi x_{\mathrm{s}}$, where $\phi$ is the correction factor. $\phi$ thus calculated lie in the range $\sim 0.16-0.24$ (Table I) and are roughly independent of composition, but depend on $x_{\mathrm{s}}$.

$\phi$ thus calculated is much lower than found earlier for polyacrylamide networks with sodium methacrylate used as ionic comonomer ${ }^{4}(\phi=1-0.97)$. There are probably several reasons for the lower $\phi$ factor found for ammonium salt: (a) Positively charged centres are localized at a larger distance from the main chain, which is probably reflected in a smaller influence on conformation. (b) Bulkiness of the ammonium group causes the electrostatic field of the positively charged nitrogen atom to be weaker compared with the electrostatic field of the carboxylic anion. Hence hydration of the ammonium group 


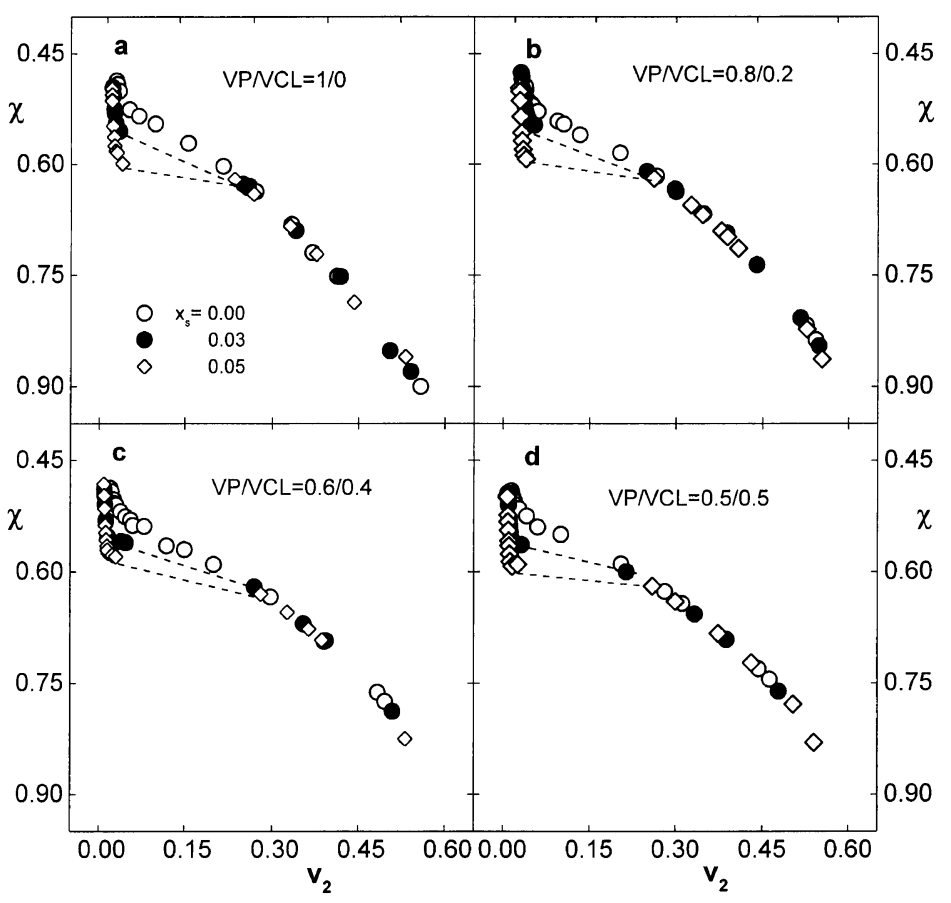

Figure 4. Dependence of interaction parameter $\chi$ on volume fraction of the dry network $v_{2}$ for indicated VP/VCL and $x_{\mathrm{s}}$ compositions. predicted jump in gel volume (eq 12).

is lower. (c) Hydration of the centers of charges is affected by counterions. For quaternary salts, the anion has the average number of hydration two, while that of the sodium cation is three. Due to this, higher hydration of electronegative centres compared with positive centres may be anticipated. (d) Differences in association of counterions for negatively and positively charged centers, and thus participation of ionic pairs. While points (a) - (c) are reflected in decrease in $\phi$, the effect of the association of counterions is difficult to discuss. Finally, this factor accounts for effects not considered in the above theory.

Using eq 11 known molecular parameters and effective ionization $i^{*}=\phi x_{\mathrm{s}}$, the dependence of $\chi$ on $v_{2}$ was calculated from $X$ measured in various water/acetone mixtures (Figure 2) for all ionized samples (Figure 4). Increase in $\chi$ on $v_{2}$ regardless of ionic salt concentration was found. Increase in $\chi$ with $v_{2}$ (with the polymer concentration in swollen polymer) was commonly observed earlier $^{1-4}$ and is related to solvent quality becoming increasingly poorer with acetone content. For ionized samples, discontinuity in $\chi$ vs. $v_{2}$ dependence associated with collapse of the gel was observed for all copolymer compositions. The example shown in Figure 5 suggests universal $\chi$ vs. $v_{2}$ dependence roughly independent of VP/VCL ratios at constant $x_{\mathrm{s}}$. As discussed previously, ${ }^{3,4}$ the van der Waals loop in the dependence of $\chi$ on $v_{2}$ is necessary for the appearance of jump change in the gel volume (1 st-order phase transition). Such dependence was found previously for many ionized networks swollen in mixed solvents at room temperature or water at various temperatures. ${ }^{4,12,16}$

From the van der Waals loop in the dependence of $\chi$ on $v_{2}$, critical interaction parameter $\chi_{\mathrm{c}}$ and compositions of coexisting phases (the values $v_{2}^{\prime}$ and $v_{2}{ }^{\prime \prime}$ ) at the transition are given by, ${ }^{3}$

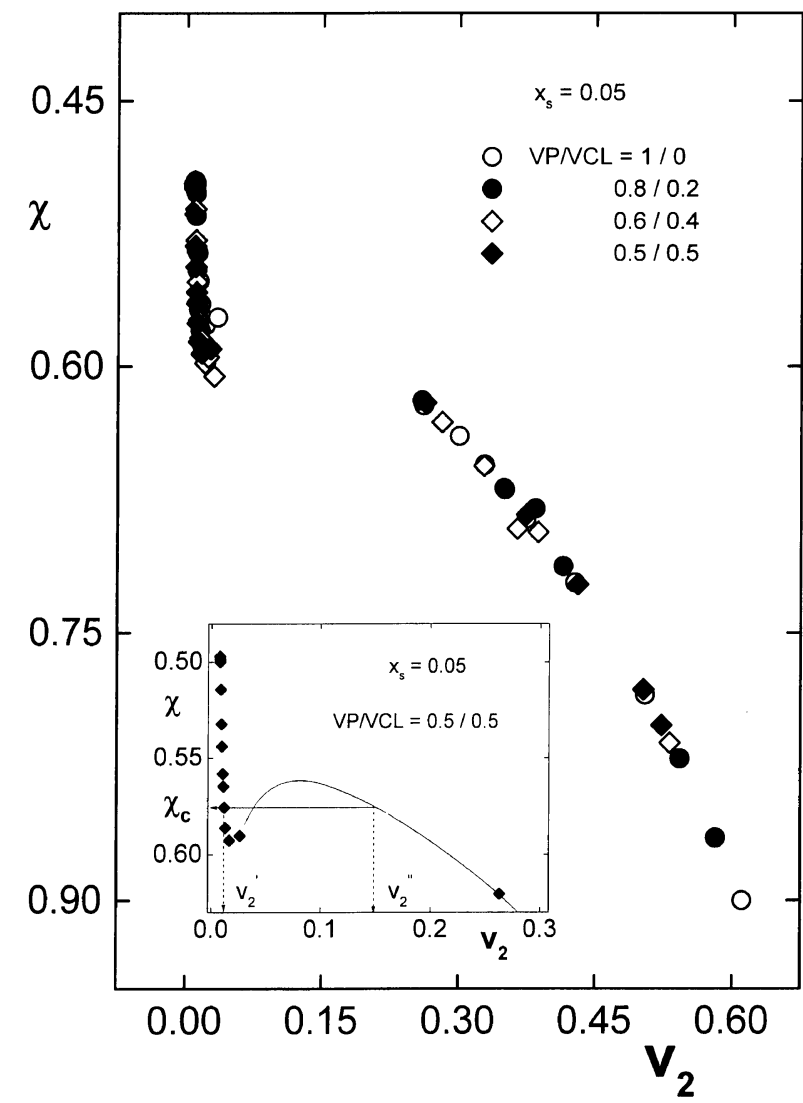

Figure 5. Example of dependence of the interaction parameter $\chi$ on volume fraction of the dry network $v_{2}$. Maxwell construction and critical parameters for network with VP/VCL $=0.5 / 0.5$ and $x_{\mathrm{s}}$ $=0.05$ are shown in inset. $\chi_{\mathrm{c}}$ and coexisting phases $v_{2}^{\prime}$ and $v_{2}^{\prime \prime}$ are presented. - course determined by eq 11 . 


$$
\int_{v_{2}^{\prime}}^{v_{2}^{\prime \prime}}\left(\chi-\chi_{c}\right) \mathrm{d} v_{2}=0
$$

In the inset in Figure 5, the van der Waals loop is shown for a network with $x_{\mathrm{s}}=0.05$ and composition VP/VCL $=$ $0.5 / 0.5$ together with eq 12 (Maxwell construction is applied to $\chi$ vs. $v_{2}$ dependence, i.e., $\chi_{\mathrm{c}}$ is determined when areas above and below the line are equal). $\chi_{\mathrm{c}}$ and extent of collapse, $\Delta_{\mathrm{t}}=v_{2}{ }^{\prime \prime}-v_{2}^{\prime}$, may thus be determined for ionized networks (Table I). $v_{2}$ vs. $a$ dependence for uncharged copolymers is known, dependence of $\chi v s . v_{2}$ can be easily transformed to dependence of $\chi$ vs. $a$ and so critical acetone compositions $\alpha_{\mathrm{c}}$ can be determined from $\chi_{\mathrm{c}}$. A comparison of the theoretically determined $\alpha_{\mathrm{c}}$ and $\Delta$ with experiment is shown in Figures $1 \mathrm{~b}$ and $1 \mathrm{c}$. The theory underestimates $\alpha_{\mathrm{c}}$ and $\Delta$. An example of theoretically determined extent of collapse $\Delta_{0.5 / 0.5}$ and position on acetone concentration for gel with $\mathrm{VP} / \mathrm{VCL}=0.5 / 0.5$ and $x_{\mathrm{s}}=0.05$ is shown in Figure 2.

The phase state of $\mathrm{P}(\mathrm{VP} / \mathrm{VCL})$ gels in water and water/ acetone mixtures is determined by interactions of polar and nonpolar groups in the chain and solvent molecules. For our uncharged networks in water, $\chi=0.492-0.498$ was found. This means that increasing hydrophobic $N$ vinylcaprolactam in copolymers only slightly decreased overall hydration of $\mathrm{P}(\mathrm{VP} / \mathrm{VCL})$ chains in pure water.

\section{CONCLUSIONS}

From swelling and mechanical measurements on VP/ VCL copolymer networks prepared by radiation polymerization, swollen in water/acetone mixtures, the following conclusions can be made:

(a) Ionic copolymer networks with required mechanical properties at high dilution can be prepared. With increasing VCL, the efficiency of crosslinking reaction decreases.

(b) Swelling of P(VP/VCL) gels in water is strongly influenced by charge on the chain and, to some extent, by VCL concentration. A comparison of experimental swelling results with theory of polyelectrolyte gels shows that agreement is reasonably good if effective ionization (i.e., corrected charge amount; correction factor $\phi$ ) is introduced.

(c) Increasing VCL in copolymers shifts the transition from expanded to collapse state to higher acetone concentrations. Gels with ammonium salt undergo firstorder phase transition. The critical acetone concentration $\alpha_{\mathrm{c}}$ and collapse, $\Delta$, increase with increasing $x_{\mathrm{s}}$. The proposed theory underestimates collapse.

(d) Jump in the modulus $G$ is correlated with jump in the swelling ratio $X$. Dependence of $\log G$ on $\log X$ is roughly the same regardless of $x_{\mathrm{s}}$ and VCL concentra- tions. Mechanical behavior is thus predominantly determined by swelling for all gels.

Acknowledgment. Financial support of Ministry of Education of the Czech Republic (Project MSM 11320001), European Commission (Grant INCOCopernicus ERBIC 15 CT 960756) and Grant Agency of the Charles University (Grants Nos 46/1998/B and 72/ 1998/B) is gratefully acknowledged. We thank Dr M. Rerichová from Artim Co., Prague, for irradiation of samples.

\section{REFERENCES}

1. K. Dušek, Ed., "Responsive Gels: Volume Transitions I and II", Adv. Polym. Sci., 109 and 110 (1993).

2. T. Tanaka, D. J. Fillmore, S. T. Sun, L. Nishio, G. Swislow, and S. Shak, Phys. Rev. Lett., 45, 1636 (1980).

3. M. Ilavský, Polymer, 22, 1687 (1981).

4. M. Ilavský, Adv. Polym. Sci., 109, 173 (1993).

5. D. De Rossi, K. Kajiwara, Y. Osada, and A. Yamauchi, Ed., "Polymer Gels. Fundamentals and Biomedical Applications", Plenum Press, New York, N.Y., 1991, pp 85-160.

6. E. A. Bekturov and Z. Kh. Bakauova, "Synthetic Water Soluble Polymers in Solution”, Huethig-Wepf Verlag, Basel, 1986, p 242.

7. M. D. Blanco, R. M. Trigo, O. Garcia, and J. M. Teijon, J. Biomater. Sci., Polym. Ed., 8, 709 (1997).

8. Y. E. Hong, T. V. Chirila, J. H. Fitton, B. W. Ziegelaar, and I. Constable, Bio-Med. Mater. Eng., 7, 35 (1997).

9. S. Senel, B. Isik-Yuruksoy, H. Cicek, and A. Tuncel, Polymer, 38, 1775 (1997).

10. N. H. Chen, T. E. Smith, and D. M. Vitus, J. Polym. Sci., Polym. Phys. Ed., 23, 461 (1985).

11. G. N. Sheth, J. Appl. Polym. Sci., 32, 433 (1986).

12. G. Mamytbekov, K. Bouchal, M. Ilavský, and E. Bekturov, Polym. J., 30, 713 (1998).

13. X. S. Wu, A. S. Hoffman, and P. Yager, J. Polym. Sci., Part A: 30, 2121 (1992).

14. R. Yoshida, K. Uchida, Y. Kaneko, K. Sakai, A. Kikuchi, Y. Sakurai, and T. Okano, Nature, 374, 240 (1995).

15. B. C. Shin, M. S. Jhon, H. B. Lee, and S. H. Yuk, Eur. Polym. J., 34, 171 (1998).

16. G. Mamytbekov, K. Bouchal, and M. Ilavský, Eur. Polym. J., 35, 1925 (1999).

17. F. Haaf, A. Sanner, and F. Straub, Polym. J., 17, 143 (1985).

18. G. B. Butler, A. Crawshaw, and W. L. Miller, J. Am. Chem. Soc., 80, 3615 (1958).

19. D. A. Topchiev, A. I. Martynenko, Yu. E. Kabanova, L. M. Timofeeva, A. S. Shashkov, and A. M. Drabkina, Vysokomol. Soedin., Ser. A, 36, 1242 (1994).

20. E. E. Makhaeva, L. T. M. Thanh, S. G. Starodubtsev, and A. R. Khokhlov, Macromol. Chem. Phys., 197, 1973 (1996).

21. K. Dusek and W. Prins, Adv. Polym. Sci., 6, 1 (1969).

22. M. Ilavský, and K. Bouchal, in "Biological and Synthetic Polymer Networks", O. Kramer, Ed., Elsevier, New York, N.Y., 1988, p 435.

23. M. Rubinstein, H. R. Colby, V. A. Dobrynin, and J.-F. Joanny, Macromolecules, 29, 398 (1996).

24. V. F. Janas, F. Rodriguez, and C. Cohen, Macromolecules, 13, 977 (1980). 